Oft werden neben Auslagerungen oder Privatisierungen Zwischenformen gewählt mit öffentlich-privaten Partnerschaften in praktisch allen Feldern öffentlicher Leistungserbringung, derzeit bei gut fünf Prozent der staatlichen Bruttoinvestitionen mit Schwerpunkten im Hochbau (Schulen und Kindertagesstätten, Sport und Kultur, Verwaltung und Justiz). Bisweilen bringen sie Zwist, etwa zwischen Regierung und Opposition im Niedersächsischen Landtag zum geplanten Bau einer Justizvollzugsanstalt. Neun Bundesländer haben im vergangenen Jahrzehnt dafür Leitstellen, Netzwerke oder Zentren gegründet. Ob die Ziele - vor allem bessere Wirtschaftlichkeit - erreicht werden, hänge, so die Autoren, stark vom Vorgehen vor Ort und der handelnden Personen ab; generelle Einschätzungen seien schwierig.

Im Blick auf die Bahnhofsdebatte in Stuttgart bietet eine vergleichende Studie zum Vertrauen in die öffentliche Verwaltung in europäischen Ländern Hilfreiches, insbesondere auch für hellhörige Abgeordnete. Befragungen zeigen, dass unter fünf Industriestaaten den Vereinigten Staaten, Deutschland, Frankreich, Großbritannien und Schweden Deutschlands Verwaltung zwischen 1981 und 2007 stets das niedrigste Vertrauen genoss. Dabei ging die Schere auseinander. In Nordamerika und Deutschland sank es, in Schweden stieg es stark. So vertrauten dort zuletzt 66 Prozent der befragten Bürger ihrer Verwaltung, in Deutschland nur 31 Prozent. Warum das aber so ist, vermochte auch diese Studie nicht zuverlässig zu erklären. Hier wäre sicher interessant gewesen zu hören, ob es eine Korrelation gibt zwischen dem Vertrauen in die Verwaltung, in die Politik, und die Parlamente.

Robert von Lucius

\title{
Abgeordnetenentschädigung: aktuell kommentiert
}

Von Arnim, Hans Herbert und Thomas Drysch: Kommentierung des Art. 48 Grundgesetz, in: Rudolf Dolzer, Wolfgang Kahl und Christian Waldhoff (Hrsg.): Bonner Kommentar zum Grundgesetz, 149. Aktualisierung, C.F. Müller Verlag, Heidelberg Dezember 2010, 162 Seiten, $€$ 349,95 (gesamtes Loseblattwerk zzgl. Aktualisierungslieferungen).

Der Zwiespalt der Loseblatt-Kommentare besteht darin, dass die Kommentierungen einzelner Bestimmungen oft umfassend sind, ja monografisch. Aber genauso oft sind sie überaltert und damit für die Beantwortung aktueller Fragen kaum noch brauchbar. Art. 48 GG über die Entschädigung der Bundestagsabgeordneten im Bonner Kommentar hatte Hans Herbert von Arnim zuletzt 1980 bearbeitet. Die Bestimmung blieb seitdem zwar unverändert, aber ihre Anwendung in der Praxis des Bundestags hat immer wieder neue verfassungsrechtliche Fragen aufgeworfen; und nachdem das Bundesverfassungsgericht im so genannten ersten Diätenurteil aus dem Jahr 1975 Art. 48 GG auch zur Auslegung der Diätenbestimmungen eines Landesparlaments heranzog, gilt sie - zu Recht oder zu Unrecht - als Maßstab auch landes(verfassungs-)rechtlicher Diätenregelungen. Für die überfällige Neukommentierung hat von Arnim seinen ehemaligen Mitarbeiter und ausgewiesenen Steuerfachmann Thomas Drysch als Koautor gewonnen. Die Kommentierung ist wiederum umfassend, aber kaum umfangreicher als zuvor; der Zuwachs an Seiten ist in erster Linie dem lesefreundlichen Satzbild geschuldet. 
Die weitgehend unveränderte Einleitung hebt die demokratische Funktion der Diäten hervor: Sie ermöglichen es allen, unabhängig von Einkommen oder Vermögen oder Zuwendungen Dritter, ein Abgeordnetenmandat auszuüben. Interessant ist die Fortschreibung der Gesetzgebungsgeschichte der Diätenregelungen in Bund und Ländern von ihrer Entstehung bis zur Gegenwart, einschließlich der zahlreichen öffentlichen Debatten um echte oder vermeintliche „Diätenskandale“. Dass die Abgeordneten „in eigener Sache“ entscheiden, ja nach Art. 48 GG entscheiden müssen, weil die Diäten durch (Parlaments-)Gesetz zu regeln sind, ist nach von Arnim und Drysch das Grundproblem der Abgeordnetenentschädigung. Sie betonen deshalb zu Recht - wie auch das Bundesverfassungsgericht - die Bedeutung der Transparenz, um eine wirksame öffentliche Kontrolle zu ermöglichen.

Viele Parlamentarier sprechen vom „Fluch“ der Entscheidung in eigener Sache, weil sie regelmäßig wegen jeder Diätenanpassung kritisiert werden, während Einschränkungen und Einschnitte oft gar nicht oder - schlimmer noch - als Bestätigung des Vorurteils viel zu üppiger Diäten wahrgenommen werden. Ein Versuch, diesem „Fluch“ zu entkommen, ist die Indexierung, die inzwischen viele Parlamente zumindest für Teile der Entschädigung vorsehen.

Von Armin und Drysch halten die Indexierung für verfassungswidrig, weil sie die öffentliche Kontrolle umgehe. Darüber kann man diskutieren: Immerhin hat das BVerfG eine Koppelung der Diäten an die Beamtenentschädigung für verfassungswidrig erachtet, weil das Parlament auf diese Weise nicht unmittelbar über die Diäten entscheide, sondern mittelbar zusammen mit der Festsetzung der Beamtenbesoldung. Ist dieser Fall aber wirklich vergleichbar mit einer Indexierung, die sich nach objektiven, vom Parlament nicht (oder allenfalls sehr vermittelt) beeinflussbaren statistischen Indikatoren richtet? Das erörtern die Autoren leider genauso wenig wie die Tatsache, dass das BVerfG in der Parteienfinanzierung die Indexierung für ein gut geeignetes Element der Steuerung und Begrenzung der Politikfinanzierung ansieht, das Thüringen und jetzt auch Bremen ausdrücklich in ihren Verfassungen verankert haben; der Thüringer Verfassungsgerichtshof hat die Verfassungsbestimmung gebilligt. Diese Entscheidung kritisieren die Autoren, legen sich aber letztlich nicht fest, ob Indexregelungen auf der Ebene der Landesverfassung gegen das Demokratieprinzip des Art. 28 Abs. 1 Satz 1 GG verstoßen und deshalb die Verfassungsautonomie der Länder überschreiten.

Während die Autoren die gegenwärtige Entschädigung der Bundestagsabgeordneten für angemessen erachten, halten sie die der Landtagsabgeordneten für zu hoch, weil sie sich (bis auf Hamburg) am Leitbild des Vollzeit-Parlamentariers orientiert. Hier bleibt die Diskussion an der Oberfläche. So bleibt unberücksichtigt, dass sich die Landesparlamente jahrzehntelang und letztlich mit einigem Erfolg für eine Stärkung der Gesetzgebungskompetenzen der Länder eingesetzt haben. Sie haben in Verfassungs- und Parlamentsreformen dem Schwund ihres Einflusses gegenüber der Exekutive Einhalt geboten. Würde die Herabstufung der Landtagsabgeordneten zu bloßen Hobby-Parlamentariern diese Entwicklung nicht konterkarieren? Und würde es dann nicht wieder vom eigenen (oder, noch schlimmer, fremden) Geldbeutel abhängen, ob man sich als Landesparlamentarier voll und ganz seinem Mandat widmen kann?

Das Bundesverfassungsgericht hatte 2000 den Kreis derer, die eine besondere Funktionsentschädigung erhalten dürfen, über die Parlamentspräsidenten und ihre Stellvertreter hinaus auf die Fraktionsvorsitzenden erweitert. Schon damals war es üblich, dass die Fraktionen aus ihren Kassen weitere besondere Funktionen in Parlament und Fraktion vergüten. 
Auch wenn das BVerfG sich zu diesen Zulagen aus Fraktionsmitteln nicht geäußert hat, halten sie die Autoren für eindeutig verfassungswidrig. Zuzustimmen ist ihnen zumindest darin, dass solche Zulagen deutlich transparenter als bisher gemacht werden sollten. Darüber hinaus kann man aber durchaus die Frage stellen, warum die Fraktionen besondere Leistungen ihrer Mitglieder, die sie im Zweifel auch extern „einkaufen“ beziehungsweise von Mitarbeitern erledigen lassen könnten (Beispiele: Geschäftsführung, Justiziariat), nicht vergüten dürfen. Vielleicht wäre es sogar sinnvoller, sämtliche Zulagen, also auch für den Fraktionsvorsitz, ausschließlich aus der Fraktionskasse zu zahlen, weil es Sache der Fraktionsautonomie ist, ob und welche Funktionen ihre Mitglieder für notwendig erachten (zum Beispiel einen oder mehrere Fraktionsvorsitzende) und ob und in welcher Höhe sie dafür zahlen wollen. Im Gegenzug wäre ein Höchstmaß an Transparenz für solche Vergütungen erforderlich, verbunden mit einer Steuerung und Begrenzung der Fraktionszuschüsse zum Beispiel - wie bei der Parteienfinanzierung - mittels einer Indexierung.

Dass steuerfreie Aufwandspauschalen von Verfassungs wegen Bestandteil der Diäten sein können, ist mittlerweile gefestigte Rechtsprechung - sofern sie sich am tatsächlichen Aufwand orientieren. Von Armin und Drysch zeigen allerdings so eklatante Unterschiede bei den entsprechenden Regelungen in Bund und Ländern auf, dass ihre Frage berechtigt ist, ob wirklich alle Regelungen diesem Maßstab gerecht werden. Einige Parlamente verzichten mittlerweile auf steuerfreie Aufwandspauschalen.

Insgesamt haben von Arnim und Drysch eine Kommentierung vorgelegt, die durch ihren Faktenreichtum und insbesondere durch die Einbeziehung der Länderregelungen besticht - auch wenn das die Gefahr mit sich bringt, dass die Reichweite des Art. 48 GG auf die Länder gelegentlich überdehnt wird. Bei dem als „Diätenkritiker“ bekannten Autor von Arnim wundert es nicht, dass Kritik an bestehenden Regelungen häufig und deutlich geäußert wird. Sie bleibt aber im Rahmen dieses Kommentars stets sachlich; und auch wenn die Argumentation gelegentlich etwas oberflächlich ist und nicht in jedem Fall überzeugt, lohnt sich die Auseinandersetzung mit ihr. Es bleibt zu hoffen, dass die nächste Neubearbeitung nicht gar so lange auf sich warten lässt.

Florian Edinger

\section{Schuldenbremse im Grundgesetz: verteidigt und fortentwickelt}

Ryczewski, Christoph: Die Schuldenbremse im Grundgesetz. Untersuchung zur nachhaltigen Begrenzung der Staatsverschuldung unter polit-ökonomischen und bundesstaatlichen Gesichtspunkten (Schriften zum Öffentlichen Recht, Band 1182), Duncker \& Humblot, Berlin 2011, 261 Seiten, $€ 58,-$.

Christoph Ryczewskis an der Humboldt Universität entstandene Dissertation greift ein tagespolitisch brisantes Thema auf. Sie untersucht die Ausgestaltung und die Defizite der seit 2009 durch die Föderalismusreform II im Grundgesetz verankerten Schuldenbremse. Die ersten circa 140 Seiten der Studie sind Bekanntem gewidmet, nämlich der Vorgeschichte dieser Reform, also den bisherigen Verschuldungsregeln auf Bundes-, Landes- und Europaebene. Deren ausführliche Betrachtung soll belegen, dass 2009 tatsächlich Reformbedarf 